The Philosophical Journal of Conflict and Violence

Vol. V, Issue $1 / 2021$

(C) The Authors 2021

Available online at http://trivent-publishing.eu/

\title{
Mapping Meaning and Purpose in Human-Robot Teams: Anthropomorphic Agents in Military Operations
}

\author{
Massimiliano L. Cappuccio, ${ }^{1}$ Jai C. Galliott, ${ }^{1}$ Eduardo B. Sandoval ${ }^{2}$ \\ ${ }^{1}$ Values in Defence and Security Technology group, University of New South Wales, Australia \\ ${ }^{2}$ Creative Robotics Lab, University of New South Wales, Australia.
}

\begin{abstract}
We spontaneously tend to project animacy and sensitivity to inanimate objects and sometimes we attribute distinctively buman features like intelligence, goals, and reasons to certain artificial devices. This phenomenon is called "anthropomorphism" and has been long studied by researchers in humanrobot interaction and social-robotics. These studies are particularly important from the perspective of recent developments in military technology, as autonomous systems controlled by AI are expected to play a greater and greater role in the future of warfare. Anthropomorphistic effects can play a critical role in tactical operations involving bybrid human-robot teams, where service members and autonomous agents need to quickly coordinate relying almost exclusively on fast, cognitively parsimonious, natural forms of communication. These forms rely importantly on anthropomorphism to allow human soldiers read the behavior of machines in terms of goals and intentions. Understanding the cognitive mechanisms that underpin anthropomorphistic attributions is bence potentially crucial to increase the accuracy and efficacy of human-machine interaction in military operations. However, this question is largely philosophical, as numerous models compete in the space of social cognition theory to explain behavior reading and mental states attribution. This paper aims to offer an initial exploration of these mechanisms from a perspective of philosophical psychology and cognitive philosophy, reviewing the theories in social cognition that are most promising to explain anthropomorphism and predict how it can enable and improve natural communication between soldiers and autonomous military technologies.
\end{abstract}

\section{Keywords}

Autonomous artificial agents; hybrid military teams; human-agent interaction; social cognition; social robotics; social psychology; situational awareness; military drone; autonomous vehicle; value-sensitive design; dogfight.

DOI: 10.22618/TP.PJCV.20215.1.139005

The PJCV Journal is published by Trivent Publishing

This is an Open Access article distributed in accordance with the Creative Commons Attribution Non Commercial (CC$B Y-N C$-ND 4.0) license, which permits others to copy or share the article, provided original work is properly cited and that this is not done for commercial purposes. Users may not remix, transform, or build upon the material and may not distribute the modified material (bttp:/ / creativecommons.org/licenses/by-nc/4.0/) 


\title{
Mapping Meaning and Purpose in Human-Robot Teams: Anthropomorphic Agents in Military Operations
}

\author{
Massimiliano L. Cappuccio, ${ }^{1}$ Jai C. Galliott, ${ }^{1}$ Eduardo B. Sandoval ${ }^{2}$ \\ ${ }^{1}$ Values in Defence and Security Technology group, University of New South Wales, Australia \\ ${ }^{2}$ Creative Robotics Lab, University of New South Wales, Australia.
}

\begin{abstract}
We spontaneously tend to project animacy and sensitivity to inanimate objects and sometimes we attribute distinctively human features like intelligence, goals, and reasons to certain artificial devices. This phenomenon is called "anthropomorphism" and has been long studied by researchers in humanrobot interaction and social-robotics. These studies are particularly important from the perspective of recent developments in military technology, as autonomous systems controlled by AI are expected to play a greater and greater role in the future of warfare. Anthropomorphistic effects can play a critical role in tactical operations involving hybrid human-robot teams, where service members and autonomous agents need to quickly coordinate relying almost exclusively on fast, cognitively parsimonious, natural forms of communication. These forms rely importantly on anthropomorphism to allow human soldiers read the behavior of machines in terms of goals and intentions. Understanding the cognitive mechanisms that underpin anthropomorphistic attributions is hence potentially crucial to increase the accuracy and efficacy of human-machine interaction in military operations. However, this question is largely philosophical, as numerous models compete in the space of social cognition theory to explain behavior reading and mental states attribution. This paper aims to offer an initial exploration of these mechanisms from a perspective of philosophical psychology and cognitive philosophy, reviewing the theories in social cognition that are most promising to explain anthropomorphism and predict how it can enable and improve natural communication between soldiers and autonomous military technologies.
\end{abstract}

\section{Keywords}

Autonomous artificial agents; bybrid military teams; human-agent interaction; social cognition; social robotics; social psychology; situational awareness; military drone; autonomous vehicle; value-sensitive design; dogfight.

\section{Introduction}

In a series of simulations recently run by DARPA as part of their "AlphaDogfight" project, artificial intelligence has systematically outperformed a team of experienced F-16 pilots, with machines winning over humans in several consecutive matches of simulated aerial 
confrontation. ${ }^{1}$ At the same time, as part of the "Loyal Wingman" project, Boeing, in collaboration with U.S. Air Force and Royal Australian Air Force, is developing a new strain of supersonic autonomous combat drones capable to fly in formation with fifth generation jetfighters controlled by human pilots (F-35), protecting them from incoming attacks and automatically coordinating with both on board computers and pilots to conduct joint missions. ${ }^{2}$ These technological advances raise new pressing questions to the researchers interested in human-computer and human-agent interaction.

Arguably, whether they are engaging in cooperative or competitive activities, human pilots need to be able to "read" the behaviour of the artificial ones in order to decode their goals, understand their tactics, and anticipate their next moves. But does the behaviour of an artificial agent look and "feel" like a human one? Are the decisions taken by a machine interpretable in an equally intuitive and effortless manner? And how can the designers and developers make sure that the behaviours of the machine appropriately display, conceal, or misrepresent the objectives pursued by the AI, to achieve the constructor's intended goals? These questions reach beyond autonomous jetfighters, as they involve the whole rapidlydeveloping domain of autonomous weapon systems.

Military analysts and foresight experts predict that the future of warfare will be shaped by the armed forces' capability to incorporate autonomous artificial agents (AAAs), i.e. synthetic systems controlled by artificial intelligence (AI) such as interactive robots, unmanned vehicles, and digital avatars. ${ }^{3}$ Whether AAAs operate in physical or merely simulated spaces, they are controlled by adaptive algorithms and machine learning systems capable to skilfully navigate and manipulate the environment, coping with contingencies in completing tasks, and even selecting the best task-resolution strategies. ${ }^{4}$

AAAs are normally classified according to varying degrees of autonomy: "from complete teleoperation, to a highly self-sufficient system that need only be supervised at the task level." 5 To comply with mission parameters and accomplish their assigned task, AAAs should be capable to autonomously set and update their local objectives and dynamically adjust their purposeful action strategies to achieve them. To do this, AAAs would need, at least, minimal understanding of the implications of their own actions. Currently, AAAs lack the cognitive prerequisites necessary to recognize relevant contextual contingencies, make sense of complex environments, and reliably interpret human priorities. AI theorists typically refer to the so called "frame problem" when they address the AI's inherent limitations in representing the contextual background of their own decisions. ${ }^{6}$

${ }^{1}$ P. Tucker, "An AI Just Beat a Human F-16 Pilot in a Dogfight - Again," Defense One online (August 20, 2020). Retrieved at https://www.defenseone.com/technology/2020/08/ai-just-beat-human-f-16pilot-dogfight-again/167872/.

2 B. Lendon, "Australian military gets first drone that can fly with artificial intelligence," CNN (2020). Retrieved at https://edition.cnn.com/2020/05/05/asia/australia-loyal-wingman-drone-intl-hnk/inde x.html.

${ }^{3}$ See P.W. Singer, Wired for War: The Robotics Revolution and Conflict in the 21 st Century (London: Penguin, 2009); P. Scharre, Army of None: Autonomous Weapons and the Future of War (New York: WW Norton \& Company, 2018); A. Swiderska, Dennis Küster, "Avatars in Pain: Visible Harm Enhances Mind Perception in Humans and Robots," Perception 47/12 (2018).

${ }^{4}$ M.L. Cummings, "Artificial Intelligence and the Future of Warfare," International Security Department and US and the Americas Programme, Chatham House - The Royal Institute of International Affairs (Jan. 2017).

${ }^{5}$ C. Breazeal, "Towards Sociable Robots," Robotics and Autonomous Systems 42/3-4 (2003): 167-175.

${ }^{6}$ M.L. Cappuccio, M. Wheeler, "When the Twain Meet: Could the Study of Mind Be a Meeting of Minds?," in Postanalytic and Metacontinental: Crossing Philosophical Divides, ed. Jack Reynolds, James Chase, Ed Mares, James Williams (London: Continuum, 2010), 125-44 ; "Ground-Level Intelligence: Inter- 
Because of these limitations, at this stage AAAs can only complement human work in military context, not replace it. In general, state-of-the-art AAAs are efficacious only insofar as human operators complement the decision-making protocols embedded in the machines with their own understanding of the mission parameters. ${ }^{7}$ Military research aims to create and manage hybrid systems composed of humans and AAAs, but their integration opens unprecedented challenges. ${ }^{8}$

Reliable, agile, and intuitive buman-agent interaction (HAI) is a fundamental prerequisite for the satisfactory functioning of hybrid teams. ${ }^{9}$ But sophisticated algorithms are not enough to establish effective HAI. ${ }^{10}$ To assure that humans and machines interface correctly and coordinate efficaciously on the battlefield, AAAs must first of all be capable to detect and correctly interpret human intentions, goals, and behaviours; not less important, in order to enable fluent interactions within teams and avoid potentially risky misunderstandings, AAAs must be able to clearly communicate the goals and the reasons of their actions to human operators. ${ }^{11}$

Hence, in order to be integrated within hybrid military teams, AAAs must be equipped with the capability to recognize other agents and be recognized as agents in turn: not only must they be able to identify meaning and purpose in human behaviour, but also produce behaviours that appear immediately purposeful, and hence meaningful, to humans. ${ }^{12}$ Humans and machines must be able to validate each other's decisions through recurrent reciprocal recognition in order to establish even simple coordination and prevent misalignments of intentions and goals that could lead to catastrophic consequences in the context of military operations.

As the operability of AAAs in warfare depends on both the humans and the machines involved, questions concerning the psychological significance and normative value of HAI are not less important than the technological details of the implementation. ${ }^{13}$ Two questions concerning the preparedness of human personnel and the user-centred design of autonomous systems are becoming central in the domain of military HAI:

(a) How do military personnel experience their interaction with artificial autonomous agents in defence and security operations?

Context Frame Problem and Dynamics of the Background," in Knowing without Thinking. Mind, Action, Cognition and the Phenomenon of the Background, ed. Z. Radman (Basingstoke: Palgrave Macmillan, 2012).

7 G. Hoffman, C. Breazeal, "Collaboration in Human-Robot Teams," in ALAA 1st Intelligent Systems Technical Conference, Infotech@Aerospace Conferences (American Institute of Aeronautics and Astronautics, 2004).

8 S.G. Hill, D. Barber, A. W. Evans, "Achieving the Vision of Effective Soldier-Robot Teaming," Proceedings of the Tenth Annual ACM/IEEE International Conference on Human-Robot Interaction Extended Abstracts - HRI'15 Extended Abstracts (2015).

${ }^{9}$ M.J. Barnes, A.W. Evans, "Soldier-Robot Teaming: An Overview," in Human-Robot Interactions in Future Military Operations, ed. M. Barnes and F. Jentsch (Farnham: Ashgate Publishing, 2010) ; M.J. Barnes, F.J. Jentsch (eds.), Human-Robot Interactions in Future Military Operations (Farnham: Ashgate Publishing, 2010).

${ }^{10}$ K. Dautenhahn, "Socially Intelligent Robots: Dimensions of Human--Robot Interaction," Philosophical Transactions of the Royal Society of London. Series B, Biological Sciences 362 (2007): 679-704.

${ }^{11}$ G. Hoffman, "Embodied Cognition for Autonomous Interactive Robots," Topics in Cognitive Science 4/4 (2012): 759-72.

12 M.L. Cappuccio, A. Peeters, W. McDonald, "Sympathy for Dolores: Moral Consideration for Robots Based on Virtue and Recognition," Philosophy \& Technology 33 (2019): 9-31.

${ }^{13}$ L. Foster Thompson, D.J. Gillan,"Social Factors in Human-Robot Interaction," in Human-Robot Interactions in Future Military Operations, ed. Barnes and Jentsch (Boca Raton : CRC Press, 2017), 67-81. 
(b) How does the design of human-machine interfaces affect the capability of a hybrid military team to successfully carry out its tasks in a collaborative fashion?

The empirical investigation necessary to answer these questions has just started and is still at an early stage. The goal of our paper is to philosophically address the theoretical and methodological foundations of such investigations, clarifying its assumptions and conceptual background.

As a preparatory step, it is useful to transfer to the military context a number of canonical concepts - such as Trust, Acceptance, and Tolerance - that are key for the methodology of social robotics and HAI research: ${ }^{14}$ Trust indicates the expectations related to the robot's intelligence and general ability to competently carry out its tasks; ${ }^{15}$ Acceptance measures how likeable and credible a robot can be as a social companion and a co-worker; ${ }^{16}$ Tolerance refers to the easiness of transferring to technology the authority and responsibility of decisions traditionally made only by humans. ${ }^{17}$ While inherently qualitative in nature, these psychological aspects of HAI must be carefully considered by engineers if they want to design efficacious applications of AI and robotics in warfare. The way AAAs are perceived by human troops makes a difference to what they can do together as part of an hybrid team, as trust, acceptance, and tolerance contribute to define the function and the scope of their interaction.

All these notions converge on a more fundamental phenomenon, called anthropomorphism. It is well documented that anthropomorphic tendencies emerge spontaneously and pervasively from HAI. ${ }^{18}$ While monitoring these tendencies is indispensable, entirely suppressing them would be neither possible nor convenient due to their pervasiveness and resilience.

This paper aims to investigate why military AAAs are often attributed anthropomorphic features and how anthropomorphism affects HAI in military teams, generating complex patterns of opportunities and risks. Unlike similar works on the same topic, our primary aim is to identify the philosophical prerequisites of a legitimate theory of anthropomorphism. To achieve this goal, first we will compare several approaches to social cognition, the discipline that studies the cognitive preconditions of social engagement. Then we will examine how these approaches have been integrated within social robotics, the interdisciplinary study of the psychological and technological factors that affect HAI. ${ }^{19}$ Finally, we will discuss how the notions and methods of social robotics research are relevant to advance the research in military AAAs.

${ }^{14}$ C. Bartneck, D. Kulić, E. Croft, S. Zoghbi, "Measurement Instruments for the Anthropomorphism, Animacy, Likeability, Perceived Intelligence, and Perceived Safety of Robots," International Journal of Social Robotics $1 / 1$ (2009): 71-81.

15 H.A. Abbass, J. Scholz, D.J. Reid, "Foundations of Trusted Autonomy: An Introduction," in Foundations of Trusted Autonomy, ed. Hussein A. Abbass, Jason Scholz, Darryn J. Reid (New York: Springer, 2018), 1-12.

${ }^{16}$ C. Breazeal, K. Dautenhahn, T. Kanda, "Social Robotics," In Springer Handbook of Robotics, ed. Bruno Siciliano, Oussama Khatib (New York: Springer, 2016), 1935-1972.

${ }^{17}$ J.C. Galliott, "The Soldier's Tolerance for Autonomous Systems," Paladyn, Journal of Behavioral Robotics 9/1 (2018): 124-36.

18 J. Reichenbach, C. Bartneck, J. Carpenter, "Well Done, Robot! The Importance of Praise and Presence in Human-Robot Collaboration," in ROMAN 2006 - The 15th IEEE International Symposium on Robot and Human Interactive Communication, 86-90 (New Jersey: IEEE, 2006).

${ }^{19}$ B. Mutlu, C. Bartneck, J. Ham, V. Evers, T. Kanda, (eds), Social Robotics: Third International Conference on Social Robotics, ICSR 2011, Amsterdam, The Netherlands, November 24-25, 2011. Proceedings (New York: Springer, 2011). 
Defense and security operations conducted by hybrid teams have offered some of the most striking and interesting case studies in the scientific literature on anthropomorphism, ${ }^{20}$ which is why civilian research in social robotics and social cognition is following closely the development of military applications of HAI. On the other hand, the outcomes of the research on anthropomorphism conducted in the fields of social cognition and social robotics should be taken into account by military research for at least two reasons: not only because ignoring the dangerous effects of anthropomorphism can lead to increased risks during hybrid military operations, ${ }^{21}$ but also because carefully considering the natural anthropomorphistic tendencies of humans may disclose new advantageous ways of integrating AAAs in military operations.

\section{Military Human-Robot Teams: the Background}

AI and robotics evolve rapidly and promise to deeply affect the way military work is organized, influencing the allocation of the relevant resources both within and amongst teams. In principle, various security and defence-related tasks can be assigned to, or conducted, with the support of AAAs: intelligence collection, surveillance and reconnaissance; infiltration and loitering for spying and sabotage; aerial assault and selective target engagement; perimeter protection; active shielding of military personnel and civilians; bomb disposal; the handling of chemical, biological and nuclear hazards; logistics and transportation; and the provision of medical and psychological assistance to military personnel, including training, team building, counselling, and conflict mediation.

The advantages offered by AAAs revolve around the elimination or mitigation of the colloquial 'dull, dirty and dangerous': reduction of the number of humans and work hours necessary to carry out a mission; minimization of the risks to human safety and integrity; a decrease to the physical, cognitive/attentional, and emotional/motivational burden necessary to carry out certain jobs, with obvious benefits for the troops' morale and cohesion; improvement in the integration of information representation, which facilitates the coordination of groups in complex scenarios; portability and exact replicability of expertise and decision-making protocols, which enables the continuation of missions despite the absence of specialized personnel. Also, as unmanned vehicles don't require life-support, they offer unprecedented opportunities for force projection in domains hardly-reachable by humans (as in underwater or stratospheric missions), extending in space and time the military's presence and its potential for agency, knowledge, and communication through physical or merely digital proxies. ${ }^{22}$

While autonomy is the quintessential property of AAAs, these systems can operate fully autonomously only in perfectly controlled environments, i.e. arbitrarily circumscribed

\footnotetext{
${ }^{20}$ J. Carpenter, “Just Doesn't Look Right: Exploring the Impact of Humanoid Robot Integration into Explosive Ordnance Disposal Teams," in Handbook of Research on Technoself: Identity in a Technological Society, edited by Rocci Luppicini (Hershey: IGI Global, 2013), 609-36.

${ }^{21}$ J. Carpenter, “Just Doesn't Look Right: Exploring the Impact of Humanoid Robot Integration into Explosive Ordnance Disposal Teams," in Handbook of Research on Technoself: Identity in a Technological Society, edited by Rocci Luppicini (Hershey: IGI Global, 2013), 609-36; P. Singer, Wired for War: The Robotics Revolution and Conflict in the 21st Century; J.C. Galliott, "Defending Australia in the digital age: toward full spectrum defence," Defence Studies 16 (2016): 157 - 175.

22 J.C. Galliott, "Unmanned Systems and War's End: Prospects for Lasting Peace," Dynamiques Internationale 8 (2013): 1 - 24; Military Robots: Mapping the Moral Landscape (New York: Routledge, 2015); "Defending Australia in the digital age: toward full spectrum defence," Defence Studies 16 (2016): 157 175 ; "The Soldier's Tolerance for Autonomous Systems," Paladyn, Journal of Behavioral Robotics 9/1 (2018): 124-36.
} 
artificial settings involving small numbers of degrees of freedom and next-to-zero unpredictable contingencies. ${ }^{23}$ Such controlled environments are rarely found in unmoderated real-life circumstances, and virtually never on a battlefield. That is why, in most real-life contexts, military AAAs are still unable to operate without human supervision. Their deployment, routine use, maintenance, fine-tuning, and customization necessarily involves the accompanying presence and directives of human operators. The expectation is that AAAs could soon be ready to participate in military missions by (partly) substituting, complementing, augmenting, or coordinating human work, whether physical or intellectual in nature, depending on their function and the kind of technology involved.

The optimization of AAAs' interactive protocols is a key factor to achieve the kind of deep and fluent integration of human and artificial work that is required to effectively employ hybrid teams in defence and security operations. To this end, the pseudo-social abilities of physical robots, unmanned vehicles, and digital avatars have been expanded to develop a capability to jointly act with humans. Such abilities include - among other things - better methods for identifying humans (e.g., face and voice recognition), interpreting their intentions, and anticipating their actions. ${ }^{24}$

Not only are social abilities indispensable to assistive applications of robotics behind the frontline, such as intelligence, logistics and training, but also to hybrid military operations on the frontline. The defense forces of the United Kingdom and Australia have recently conducted a large-scale exercise as part of a concerted effort undertaken by the 'Five Eyes' (FVEYs) intelligence community. This exercise, called "Autonomous Warrior," was carried out in Jervis Bay, New South Wales, in June-July 2018 and in Salisbury Plain, south of England, in January 2019. Unclassified information about the logistical preparation, scale, and general structure of these exercises confirms the crucial importance attributed by military commands to hybrid teams. ${ }^{25}$ In the exercise performed by the British Armed forces, more than 50 unmanned systems from industry were deployed to complete similar tasks. ${ }^{26}$

As expected, some of the challenges faced in the attempt to achieve optimal coordination between humans and machines were technical in nature: for example, to "prevent jamming and disruptive interference," when a high number of autonomous systems were simultaneously communicating with one another and the human operators, and ensuring that "the control systems do not interfere with each other" due to the "increasingly crowded radiofrequency spectrum, especially as several unmanned systems jostle for space to beam back high-resolution data." 27

But the greatest challenges are arguably those involving the quality of human-machine communication. Normally, machines are designed to communicate using formal protocols to share information encoded in a symbolic language. This significantly constrains any possibility to establish natural, fluid, semantically rich, and effortless interaction with humans, engendering critical problems in the space of tactical operations. The design of AAA informed by human-centred principles can offer a solution to this problem.

\footnotetext{
${ }^{23}$ M.L. Cappuccio, M. Wheeler, "When the Twain Meet: Could the Study of Mind Be a Meeting of Minds?," in Postanalytic and Metacontinental: Crossing Pbilosophical Divides, ed. Jack Reynolds, James Chase, Ed Mares, James Williams (London: Continuum, 2010),125-44.

${ }^{24}$ K.A. Cosenzo, M.J. Barnes, "Human-Robot Interaction Research for Current and Future Military Applications: From the Laboratory to the Field," Unmanned Systems Technology XII (2010).

${ }^{25}$ G. Slocombe, "Autonomous Warrior: Major Air, Land and Sea Exercise," Asia-Pacific Defence Reporter (September 26, 2019).

${ }^{26}$ G. Turnbull, "Why the British army tested robots in muddy fields," C4ISRnet (January 11, 2019).

${ }^{27} \mathrm{Ibid}$.
} 
That is why the Australian armed forces created and tested software to better manage the so-called "fog of war". The software advises the units involved in complex operations "using virtual human characters" 28 to provide real-time information and increase situational awareness. Interestingly, the software uses humanoid digital avatars that speak and act in a simulated environment. The choice to give an anthropomorphic look to this AAAs is significant, Other, more impersonal, options would have been immediately available and easier to implement - for example, representing information in a detached and neutral format that includes only text and graphs. Evidently, the designers found no reason to worry that the avatar's humanoid look could represent a distraction or a source of risky behaviours (in the hypothetical case that troops stopped seeing the system as a tool and start treating it as a person).

In fact, despite these potential complications, the developers have deliberately given emphasis to the embodied and narrative character of the AAA, in an attempt to exploit its role as humanoid informer, coordinator, and supervisor. This choice aims to make communication with human agents more spontaneous and natural, overcoming the problematic lack of fluency and richness in HAI by exploiting the advantageous effects of anthropomorphism during the design stage. ${ }^{29}$

\section{Anthropomorphism: the Theory}

The attention to the anthropomorphic design of military AAAs confirms that the effects of anthropomorphism are taken seriously by the military researchers, who seem interested in how the warfighter's attentional, behavioural, and emotional responses are modulated by natural interaction with AAAs during hybrid operations. That is why it is useful to briefly introduce the notion of anthropomorphism and discuss its practical implications.

There are at least two different definitions of anthropomorphism. ${ }^{30}$ According to the most common definition, anthropomorphism is the tendency to attribute to non-human entities the characteristics of human ones, transferring to the former psychological attitudes and forms of engagement that are typically reserved to the latter. Zoomorphism is a phenomenon closely related to, and partly overlapping with, anthropomorphism, as it identifies the tendency to attribute to inanimate entities the features of animals or animal-like entities (including the homo sapiens animal species). Among the characteristics that can be projected onto a non-human, possibly inanimate, entity there are both basic features (goal-oriented, adaptive behaviour and the capability to selectively sense and track contextually relevant cues) and more cognitively complex features like knowledge states (beliefs), objectives (desires), and plans to achieve them (intentions), which arguably involve propositional and semantic content.

Social robotics research has highlighted that behavioural anthropomorphism correlates to higher levels of likeability and perceived intelligence of robot, which, in turn, predict more resilient trust and robust interest. Note that different groups of humans (for example autistic and typically developing children) show significantly different preferences about the kinds of

28 G. Slocombe, "Autonomous Warrior: Major Air, Land and Sea Exercise."

${ }^{29}$ K.A. Cosenzo, M.J. Barnes, "Human-Robot Interaction Research for Current and Future Military Applications: From the Laboratory to the Field."

30 J.A. Złotowski, H. Sumioka, C. Bartneck, S. Nishio, H. Ishiguro, "Understanding Anthropomorphism: Anthropomorphism Is Not a Reverse Process of Dehumanization," in Social Robotics, ed. Abderrahmane Kheddar, Eiichi Yoshida, Shuzhi Samk GeKenji Suzukik John-John Cabibihan, Friederike Eyssel, Hongsheng He (NY: Springer International Publishing, 2017), 618-27. 
robots with which they like to engage. ${ }^{31}$ For some humans it is reasonable to have a robot as a companion or even as an intimate mate, while for others the very idea of treating a robot like a person sounds disturbing. Such individual variations should be accounted for in the development of hybrid military teams, as AAAs should ideally be able to detect them and adjust their behaviour accordingly.

The cognitive/behavioural mechanisms underpinning the anthropomorphistic experience are very deep, developmentally early, most probably innate, and related to the instinctive tendency to attribute animacy and intentions even to entities that have little or no resemblance at all to animated or living creatures. The fact that anthropomorphism is hardwired in human cognition is compatible with the conjecture that it scaffolds some of our skills in social and cultural cognition. That is why various cognitive biases and delusions arise from a background of anthropomorphic attributions and personification. ${ }^{32}$

Anthropomorphism is not always intentional and explicit. Rather, it often emerges at a pre-reflective level, embedded in a set of preferences or subconscious behavioural dispositions. That is why researchers emphasize that the phenomenon of anthropomorphism can be either implicit or explicit: it is implicit when a subject pre-reflectively responds to an inanimate entity as if it was animated, attesting with their behaviour or conduct the attribution of animacy or agency; we call explicit the anthropomorphic attributions expressed through verbal behaviours. ${ }^{33}$ The former can operate automatically, at the level of fast, immediate perceptual and habitual responses of which the agent might not be mindful of, while the latter operates through conscious, reflective control, at the level of judgement and rational evaluation. Explicit anthropomorphism may arise from the deliberate search of human-like features - for example, as part of the process of personification of nature that is at the root of figurative or poetic creation.

Explicit and implicit attributions are semi-independent and not necessarily consistent with each another: it is well possible to immediately anthropomorphize a robot at the perceptual level (for example when its movement show zoomorphic biological and features) while holding the belief that robots are just unintelligent inanimate entities; on the other hand, it is possible to judge a software as truly intelligent and autonomous while feeling completely detached from its immediate behavioural expressions.

It would be impossible to make sense of our complex relationship with AAAs without considering the fundamental distinction between implicit and explicit anthropomorphism, and their semi-independent levels of cognitive elaboration. The correct identification of these different levels and the definition of their specific function within an overarching cognitive architecture is one of the goals of social cognition.

\section{Anthropomorphism: the Underlying Psychological Mechanisms}

Research in social cognition primarily focuses on the mechanisms and processes that enable the identification of other cognitive agents as cognitive agents, and the interpretation of their behaviours and mental states. Anthropomorphism is a pivotal issue for social cognition

\footnotetext{
31 A. Waytz, J.T. Cacioppo, N. Epley, "Who Sees Human? The Stability and Importance of Individual Differences in Anthropomorphism," Perspectives on Psychological Science 5/3 (2014): 219-232 ; G. Airenti, "The Development of Anthropomorphism in Interaction: Intersubjectivity, Imagination, and Theory of Mind," Frontiers in Psychology 9 (November 2018): 2136.

32 M. Dacey, “Anthropomorphism as cognitive bias," Philosophy of Science 84/5 (2017):1152-1164.

33 J.A. Złotowski, H. Sumioka, F. Eyssel, S. Nishio, C. Bartneck, H. Ishiguro, "Model of Dual Anthropomorphism: The Relationship Between the Media Equation Effect and Implicit Anthropomorphism," International Journal of Social Robotics 10 (2018): 701-714.
} 
research because the perception of anthropomorphic features strongly predicts social responses, and social engagement in turn requires at least some minimal form of anthropomorphic perception. ${ }^{34}$ Perceiving an agent as anthropomorphic is a necessary nonsufficient condition for conducting meaningful social interaction with it, as engagement can acquire a truly "social" colour only when it reciprocally connects agents that are (or seem) alive.

Numerous explanations of anthropomorphism have been provided by the main theories that compete in the arena of social cognition. ${ }^{35}$ It is useful to distinguish here between cognitivist and embodied approaches to social cognition, and their different takes on anthropomorphism. ${ }^{36}$ The cognitivist approaches intend social cognition as an inferential and representational process, mostly based on the manipulations of internal models of the others' mental states in accord with abstract rules or a priori principles; the embodied approaches understand social cognition as know-how, i.e. the ability to engage social agents through perception and action for establishing meaningful patterns of responses based on analogy or reciprocity.

Cognitivist approaches like Theory of Mind (ToM) build on inferentialist and representational principles. According to ToM, social interaction is effective when we are able to guess the other's mental contents. This guessing is possible because we use folk psychological knowledge to generate a meta-representational model of the other agent's internal representational states. The proponents of TOM believe that such models can infer when and in what form a certain mental state arises using general logical correlations between mental states and the bodily behaviours that cause them. ${ }^{37}$

Like ToM, also Intentional Stance Theory (IST) is a cognitivist approach to social cognition that explains anthropomorphism as attribution of high-level mental states like beliefs, intentions, and desires to an agent on the basis of a rational inference to the best explanation: the mental state to be attributed to the agent is the one that best fits the circumstances of the perceived action according to a rational model of optimality and efficiency. On the other hand, the Teleological Stance Theory (TST) is a deflationary version of IST. While not explicitly embodied, this approach tries to do without the cognitivist assumptions of its counterpart: TST presupposes that the inference to the best explanation operates at a level more fundamental than propositional knowledge, as it replaces the triangular correlations between desires, intentions, and beliefs presupposed by IST with an equivalent set of correlations between perceived goals, actions, and environmental conditions, respectively. ${ }^{38}$

At the opposite side of the spectrum, the theories of empathy understand anthropomorphism as an embodied process based on internal simulation and projective processes of bestowal/attribution: familiar psychophysical correlations that belong to one's own

\footnotetext{
${ }^{34}$ G. Airenti, “The Development of Anthropomorphism in Interaction: Intersubjectivity, Imagination, and Theory of Mind."

35 A. Waytz, N. Epley, J.T. Cacioppo, "Social cognition unbound: Insights into anthropomorphism and dehumanization," Current Directions in Psychological Science 19/1 (2010) :58-62 ; E.G. Urquiza-Haas, K. Kotrschal, "The Mind behind Anthropomorphic Thinking: Attribution of Mental States to Other Species," Animal Behaviour 109 (November 2015): 167-76.

36 S. Gallagher, "Understanding Others: Embodied Social Cognition," in Handbook of Cognitive Science, ed. Paco Calvo and Antoni Gomila (San Diego: Elsevier, 2008), 437-52 ; "Inference or Interaction: Social Cognition without Precursors," Philosophical Explorations: An International Journal for the Philosophy of Mind and Action 11/3 (2008): 163-74.

${ }^{37}$ G. Airenti, "The Development of Anthropomorphism in Interaction: Intersubjectivity, Imagination, and Theory of Mind."

38 M.L Cappuccio, "Inference or Familiarity?," Synthesis Philosophica 58/2 (2015): 253-72.
} 
experiential repertoire are attributed to another on the basis of an analogical process. ${ }^{39}$ "Empathy" is an English term derived from the German "Einfühlung" (feeling inside or through another's body). ${ }^{40}$ Empathy is often considered a key component of social expertise and an enabler of altruistic behaviour, but in its original phenomenological formulation it specifically refers to one's ability of perceiving the other's body as alive and sensible, based on an analogical pairing ("Paarung") between the former and the latter.

The idea behind empathy is that every cognitive agent is familiar with the history of its own emotional and intentional states, and has an intuitive knowledge of how those states correspond to specific behavioural expressions, it tends to automatically attribute the same emotions and intentional states to other agents when they display similar behavioural expressions. The very recognition of other cognitive agents as cognitive agents is based on an embodied analogy with oneself, as it involves transferring one's own cognitive and phenomenological states to another's body. This analogical link explains our capability and tendency to use our experiences of social interaction with conspecifics as standard templates to articulate rich narrations about the non-social world. This is how empathy allows us to assign intelligible meanings to natural events, personifying them.

Interaction theory represents a different embodied approach and positions itself as an alternative or perhaps a supplement to the theories of empathy: from an interactionist perspective, what allows two or more cognitive agents to mutually understand their intentions, align their goals, and recognize one another as cognitive agents is the fact that their behaviour is from the beginning determined by the context of reciprocal interactions in which they meet one another, and by the recurrent patterns of solicitations and responses that are enacted by said agents to make sense of their recurrent interaction. ${ }^{41}$

Ecological psychology can be considered a complement to the embodied approaches, as it understands anthropomorphism as a way of reducing the complexity of perceptual contingencies using only a relevant set of social affordances: we make sense of complex scenarios by reading them in terms of landscapes of action opportunities offered to both ourselves and other agents through the spatial environment we share. Seeing the behaviour of an AAA as purposeful and human-like involves the implicit solicitation of the practical know-how that is required for dealing with familiar intersubjective contexts in which social actions are typically afforded by with the help of other agents.

Cognitivist and embodied theories attempt to explain anthropomorphic attributions using different psychological principles. That is why they inevitably focus on different sets of cognitive resources: the latter relies on experiential accounts of others' behaviours based on familiarity or habit; the former on general inferential principles derived from abstract psychological models (based on either logical deduction or empirical induction) of the other minds.

${ }^{39}$ V. Gallese, "The Shared Manifold Hypothesis: Embodied Simulation and Its Role in Empathy and Social Cognition," in Empathy in Mental Illness, ed. Tom F. D. Farrow, Peter W. R. Woodruff, Tom F. D. Farrow, and Peter W. R. Woodruff (Cambridge: Cambridge University Press, 2007), 448-72.

40 See G. Airenti, "The Cognitive Bases of Anthropomorphism: From Relatedness to Empathy," International Journal of Social Robotics 7/1 (2015): 117-127; M.A. Harrison, A.E. Hall, "Anthropomorphism, Empathy, and Perceived Communicative Ability Vary with Phylogenetic Relatedness to Humans," Journal of Social, Evolutionary \& Cultural Psychology 4/1 (2010): 34 ; L.D. Riek, T. Rabinowitch, B. Chakrabarti, P. Robinson, "How Anthropomorphism Affects Empathy toward Robots," in 2009 4th ACM/IEEE International Conference on Human-Robot Interaction (HRI) (2009): 245-46.

41 S. Gallagher, "Inference or Interaction: Social Cognition without Precursors." ; G. Airenti, "The Development of Anthropomorphism in Interaction: Intersubjectivity, Imagination, and Theory of Mind." 
The embodied approaches, whether based on interaction or simulation, are better positioned to account for the phenomenon of anthropomorphism as it emerges in the military applications of HAI. As the embodied approaches describe automatic low-level responses that are built in one's pre-reflective perceptual and practical dispositions and don't require explicit cognitive effort, they are able to capture the specificity of fast-paced, emotionally charged, and practically characterized situations, such as the tactical operations in which AAAs are anthropomorphized by service members.

Arguably, while these two groups of theories differ in multiple ways, it is possible to combine them in a pluralistic epistemological account of anthropomorphism. Not only do embodied and cognitivist approaches to social cognition offer different explanations of anthropomorphism; they also describe two distinct levels or manifestations of the same anthropomorphic phenomenon. An explanatory account that integrates these two levels can dissolve the apparent contradiction that characterizes the anthropomorphic experience in the military context: military professionals can and often do attribute anthropomorphic features to AAAs (and, occasionally, they seem able to develop attachment and moral consideration for them), even if they perfectly know, at the rational level, that robots and AIs are just mechanical devices devoid of emotional states, proper motivations, and purposes. Dissolving this paradox will help us make sense of the cases of anthropomorphism documented by the literature on military HAI.

\section{Weaponizing Anthropomorphism: the Risks}

Military personnel, even very qualified ones, can become psychologically close to robots when they work together for long periods in conditions of high stress. Service members have in some cases developed anthropomorphistic responses and mild forms of attachment for the robots that assisted them in their missions, even though said robots look barely human or animal-like. Namely, explosive ordnance disposal robots were assigned names and gendered identities by the soldiers who worked with them in Iraq and Afghanistan. When one of these robots was damaged, its loss was not simply grieved like the destruction of an expensive piece of equipment, but like the death of a teammate. Singers reports that, when one of these robots was sent to the headquarters for reparation after suffering structural damage, its "teammates" requested that its mechanical parts were not replaced, but accurately fixed to preserve the individual identity of the robot. ${ }^{42}$ In at least one documented case, one soldier risked his own life to save a robot from enemy fire.

Since robots are obviously not sentient, the attachment developed by military personnel to their robots has been typically deemed as an irrational behaviour. As such, it looks incompatible with the notion that military personnel trained for operating AAAs are wellinformed about the nature of their artificial partners and act responsibly in accord with strict safety protocols. Accordingly, Carpenter has hypothesized that anthropomorphic tendencies for an AAA (namely, empathic responses including attachment) could lead to counterproductive or even risky behaviours. ${ }^{43}$ For example, if personnel feel subconsciously compelled to protect the safety of AAAs as if they were actual teammates. Consequently, Carpenter recommends caution in designing ordnance disposal robots, as their anthropomorphic look (especially now that developers are focusing on biped models), might trigger an even stronger attachment in human users.

\footnotetext{
${ }^{42}$ Singer, Wired for War: The Robotics Revolution and Conflict in the 21st Century.

${ }^{43}$ J. Carpenter, "Just Doesn't Look Right: Exploring the Impact of Humanoid Robot Integration into Explosive Ordnance Disposal Teams."
} 
However, our implicit/explicit distinction, drawing a separation between embodied and cognitive levels of social cognition, motivates a different kind of approach. Attachment for robots does not necessarily have to be considered an irrational behaviour. It originates at the level of cognitive processing - the level of embodied, affective intelligence - that is more basic and fundamental than detached, inference-based, rational judgment but does not necessarily compete with it. In fact, embodied responses may well contribute to scaffold the kind of situated intelligence and adaptive responses that constitute the concrete background of practical rationality.

Accordingly, anthropomorphism for military robots should neither be considered a failure of the troops' capabilities of rational evaluation, nor simple as the "metaphorical" attribution of features. The first hypothesis, ${ }^{44}$ fuelled by the belief that anthropomorphism is a dangerous effect of HAI, is wrong because it does not acknowledge that both the implicit and the explicit level of cognition are constitutive components of the normal human capability to critically evaluate complex situations, as often arise in military team operations, which arguably involve both emotional/embodied intelligence and detached judgment. The second hypothesis (epitomized by Barnes \& Evans 2010), fuelled by the belief that anthropomorphism is just a derivative, symbolically mediated construction enabled by linguistic/cultural representations, is also wrong because it fails to acknowledge that any explicit process of personification is scaffolded by an implicit anthropomorphic process, and hence is deeply rooted into the human's embodied expertise, emotional engagement, and perceptual responses.

Both views fail to recognize that anthropomorphism is a constitutive component of HAI, not its by-product, because embodied social cognition abilities are a fundamental requirement of intelligent decision-making and adaptive behaviour during intersubjective coordination. Hence, the function of anthropomorphism is neither intrinsically negative nor derived from cultural or linguistic representations. While the risks associated with dysfunctional HAI are not to be underestimated, evidence suggesting that anthropomorphism leads to risky behaviours is scarce, merely anecdotal, and cannot justify a general ban of anthropomorphic design. Furthermore, even if inappropriate attachment for robots occurred systematically, its risky effects could be easily countered by appropriate monitoring and training.

The practical advantages offered by a responsible implementation of anthropomorphic features in a military robot's design will often surpass the potential risks when understood along the above-mentioned lines. Anthropomorphic effects are actively exploited by assistive robotics for their positive effects in civilian (e.g., domestic, clinical, educational) contexts. ${ }^{45}$ The differences between civilian and military applications of interactive robots should not be overlooked, ${ }^{46}$ but considering the potential advantages of anthropomorphism for military HAI is more promising than looking only at poorly documented risks. In fact, as we will argue through the rest of the paper, anthropomorphic tendencies can be deliberately elicited and controlled to improve human-machine interface, creating more reliable and efficient communication between troops and robots.

\section{Weaponizing Anthropomorphism: the Tactical Applications}

Intuitive communication methods to control autonomous and semi-autonomous agents have been tested in the context of military research. To ensure an accurate and intuitive

\footnotetext{
${ }^{44}$ See ibid.; Singer, Wired for War: The Robotics Revolution and Conflict in the 21st Century.

${ }^{45}$ L. Damiano, P. Dumouchel, "Anthropomorphism in Human-Robot Co-Evolution," Frontiers in Psychology 9 (2018): 117.

${ }^{46}$ J.C. Galliot, "Uninhabited systems in the civilian realm: Some ethical concerns," IEEE Technology and Society Magazine 31 (2012): 13-16.
} 
coordination between AAAs and humans, such communication methods can involve embodied cues like hand gestures (which provide an intuitive control method based on iconic semantics ${ }^{47}$ ), gaze direction (to establish join attention and facilitate natural action coordination), and emotional expressions (through voice or facial expressions).

Natural communication methods offer significant operational advantages in HAI. ${ }^{48}$ Intuitive control does not require long and demanding training and frees up cognitive and physical resources (memory, attention and bodily engagement) that the human agent should preferably allocate to other tasks, for example communication tasks that require eye contact or bimanual manipulative tasks (like operating a computer).

Multi-tasking can and must be carried out fluidly and efficaciously, but this is possible only if the concurrent tasks don't interfere with one another, competing for the same type of cognitive resources. ${ }^{49}$ That is why cognitive parsimony is particularly important for military operations that require efficient decision-making and prompt reactions to situational changes, with significant demands on working memory and attentional resources.

Intuitive/natural control is advantageous because favours cognitive parsimony in a specific subset of tactical contexts, that we call hot scenarios. To circumscribe this subset, we must distinguish it from cold scenarios. We call "hot" those emotionally charged, risky, hightempo, scenarios that involve direct embodied (perceptual, motoric) engagement, prioritize agency over critical analysis and planning, require quick decisions of a practical nature, readiness to generate short-term predictions, and the ability to fluidly transition from one context of action to another. Cold scenarios, in turn, involve timeframes sufficiently relaxed to enable analytic and critical approaches to problem-solving, detached and disembodied form of control, a spectatorial stance, and low direct risks for the cognitive agent. Hot scenarios can be found in active combat operations, while cold ones correspond to intelligence collection tasks or peacetime activities that involve planning and strategic management and are typically conducted while sitting at a desk or behind a computer.

Anthropomorphism is unlikely to impact significantly on cold scenario (for example, when human operators are required by their role to produce neutral offline judgment and detached scrutiny) because implicit responses are less likely to arise when HAI is not based on direct perceptual engagement dynamics. On the contrary, tactical operations conducted by warfighters typically involve hot scenarios calling for value-laden decisions. When such operations are carried out by hybrid-teams comprised of humans and AAAs, anthropomorphism is a factor to be considered carefully as it might significantly impact on the appropriateness of human decisions and the chances of success of the whole team. This is because the warfighter must rely on their perceptual knowledge to make fast and effective decisions, having been trained to navigate uncertain contingencies using reflexive behaviour.

Team coordination is quick and trusted when it relies on a spontaneous, intuitive two-way signalling activity. Even when coordination among professional operatives relies on strict protocols acquired during training (rules of engagement, codes of communication, and deontological principles that soldiers have internalized), the actual implementation in real-life

\footnotetext{
47 See M. Obaid, F. Kistler, G. Kasparavičiūte, A.Y. Yantaç, M. Fjeld, "How Would You Gesture Navigate a Drone?: A User-Centered Approach to Control a Drone," in Proceedings of the 20th International Academic Mindtrek Conference (New York: Association for Computing Machinery, 2016), 113-21.

${ }^{48}$ T. Ono, M. Imai, H. Ishiguro, "Anthropomorphic Communications in the Emerging Relationship between Humans and Robots," Proceedings 9th IEEE International Workshop on Robot and Human Interactive Communication (2000).

${ }^{49}$ J.Y. Chen, S. Quinn, J. Wright, M. Barnes, D. Barber, D. Adams, "Human-Agent Teaming for Robot Management in Multitasking Environments," 2013 8th ACM/IEEE International Conference on HumanRobot Interaction (HRI) (2013).
} 
situations necessarily involves the ability to intuitively detect intentions and emotions in the others (both teammates and adversaries) and quickly adjust to their conduct.

Reading others' behaviours and emotional expressions (pain, fear, impatience, intention to maintain one's position or flee, disposition to open fire or wait) is done automatically and is considered part of a natural set of social abilities, but appropriate training can help channel one's embodied know-how to maximize its efficacy in well-coordinated and planned tactical scenarios. It is of course likely that the natural ability to read behaviour and emotion are hindered by contingent factors: when obfuscated by medical or psychological injuries, when confounded by differences in culture or code of behaviour, when the information from multiple concurrent sources is overwhelming and slows down the process of reciprocal coordination, when the nature of others' intentions is opaque due to deceptive intentions or the intrinsic limits of bodily expressivity.

The last case importantly includes the challenges associated with the interpretation of human bodily actions that use machines or artificial tools as medium. Interpreting the mental state of a soldier in flesh and bone through her gestures and facial expressions is often easier than interpreting the same mental states when the soldier is inside a car, tank, airplane, if the vehicles hides her bodily expressions. Our normal ability to perceive and interpret the others' intentions largely depends on how automatic sensorimotor responses and reciprocal expectations are established through embodied intersubjective coupling, i.e. a learning process in which two or more agents reciprocally re-define their ways to meaningfully act upon and perceive the world.

Anthropomorphism theory must and can explain how inanimate objects can mediate intersubjective coupling, acting as proxies for embodied interaction, when the physical or symbolic properties of the object evoke intentional actions enacted by living agents with their familiar expressive states. When this happens, even simple geometric shapes, with their neutral patterns of bi-dimensional movements, can near-instantaneously convey anger, or fear, or intentions like "fleeing," "capturing," "hiding from" to one or more individuals or others artificial agents operating in a team.

\section{Cars, Drones, and Tanks with an Attitude}

An exemplification of this general psychological notion is offered by how different styles of car driving can convey information about the driver's intentions, goals, and emotional states. ${ }^{50}$ All of us, and especially the most expert drivers, can intuitively read another driver's intentions through their car's movements. Knowing that the car does not have intentions of its own does not prevent us from seeing the driver's intention through the car's movements. ${ }^{51}$ Anthropomorphic cues are typically not processed as an explicit set of syntactic rules or inferential principles, but as implicit perceptual know-how. As this process is automatic, the observer does not necessarily know how it works, and yet may be able to perfect it and make it more efficacious with appropriate training and expertise development.

Now, if anthropomorphic meanings bleed through the perceived behaviour of cars driven by human drivers, so that a certain driving style can betray the broad horizon of reasons and motivations that characterize a human driver's decisions, then the behaviour of self-driving cars should be anthropomorphized in the same way if it uses the same driving style. The

\footnotetext{
${ }^{50}$ R. Sparrow, M. Howard, "When Human Beings Are like Drunk Robots: Driverless Vehicles, Ethics, and the Future of Transport," Transportation Research Part C: Emerging Tecbnologies 80 (July 2017): 206-15. ${ }^{51}$ J.Y. Chen, C. Anthony, R. Selkowitz, K. Stowers, S.G. Lakhmani, M.J. Barnes, "Human-Autonomy Teaming and Agent Transparency," Proceedings of the Companion of the 2017 ACM/IEEE International Conference on Human-Robot Interaction - HRI '17 (2017).
} 
behaviour of self-driving cars is very likely to reflect fine-grained patterns of human-like intentional behaviours captured through the statistical analysis of myriads of videoclips of human drivers. 52

If, through machine learning technologies and appropriate fine-tuning by human trainers, autonomous vehicles (including cars, tanks, or aircraft) can reproduce the increasing number of patterns from the wide horizon of intentional patterns that characterize the human driving activity, then it is reasonable to assume that human observers, and perhaps also other machines, can detect these patterns and extract useful information to explain the car's behaviour, interpret its causes in terms of motivations and reasons, or anticipate its effects, characterizing them as goals and desires.

When sufficiently fine-grained, this information can be crucial in hot situations, as it allows the observer to quickly adjust to the car's behaviour and coordinate with it. In turn, the complete lack of such information could be misleading for a human observer strongly used to rely on the intentional information communicated by the car's behaviour to adjust its own conduct at the wheel. ${ }^{53}$ Confusion could arise if autonomous vehicles were programmed to display only psychologically neutral and mechanical behaviours, filtering out the finegrained details that suggest emotion or intention.

Now let us imagine that human drivers are expected to coordinate with unmanned vehicles to accomplish a certain collaborative task, such as driving in formation to escort a military convoy. Could the dearth of anthropomorphic information expressed by unmanned vehicles disrupt the overall performance of the hybrid team? Or should we rather think that anthropomorphic information is merely aesthetic and cosmetic, but does not play any functional role in human-machine interaction? The empirical evidence necessary to answer this question is sparse, but we can rely on the well-established practical know-how and common sense embedded in the practices and mindset of technology designers. Lay evidence suggests that humans already perceive the intent of low-level autonomous vehicles and rely on their evolving recognition of the relevant patterns in transitioning from a non-autonomous to highly autonomous transport future.

Car designers and marketers also know that vehicles have a "body," a "face," "eyes," and that these elements contribute to shape the perceived personality of the car and hence attract different types of potential buyers. ${ }^{54}$ This suggests that aesthetics and functional design can and often are strongly intertwined. We also know that human-human interaction massively relies on tenuous behavioural cues of which we typically are barely aware of (body language, voice intonation, gaze direction, etc.), and that the accuracy of explanatory/predictive behaviours (whether in cooperative or competitive contexts) strongly depends on the expectations formed by human agents on these cues.

Hence, coordination of complex activities involving manned and unmanned vehicles could be affected by the way anthropomorphic information is or is not conveyed by the unmanned vehicles, and by the reciprocal capability of unmanned vehicles and human operators to correctly interpret each another's anthropomorphic cues in terms of intentional and emotional states. This consideration is even more significant in the context of interactions

\footnotetext{
52 S. Nyholm, "The Ethics of Crashes with Self-Driving Cars: A Roadmap," Philosopby Compass 13/7 (2018).

53 Ibid.

54 C. DiSalvo, F. Gemperle, "From Seduction to Fulfillment: The Use of Anthropomorphic Form in Design," in Proceedings of the 2003 International Conference on Designing Pleasurable Products and Interfaces (2003): 67-72 ; C. DiSalvo, B. Hanington, J. Forlizzi, "An Accessible Framework of Emotional Experiences for New Product Conception," in Design and Emotion, ed. Deana McDonagh, Paul Hekkert, Jeroen van Erp, Diane Gyi (Boca Raton: CRC Press, 2003).
} 
between unmanned military vehicles, such as autonomous tanks, underwater vehicles, and flying drones of varying size and role. This importantly includes, as mentioned in this paper's Introduction, competitive interactions with new generation drones excelling in dogfight, in which the drone's ability to produce a certain intentional behaviour and the human pilot's ability to correctly and quickly read that behaviour may eventually decide which party will win the aerial confrontation.

\section{Conclusions}

Recognizing and controlling the way military personnel subconsciously project animacy, intentionality, agency, and purpose on AAAs is not just a way to discipline their relationship, but also a necessity for the success of the team as a whole. Anthropomorphizing these devices can be a cognitively parsimonious, operationally efficacious, and properly rational way to make sense of their behaviours and successfully coordinate with them to complete joint tasks. This is especially true of 'hot' (tactical) scenarios that require agents to directly establish interactions of an embodied, perceptual nature and to make quick decisions utilizing personcentred, highly context-sensitive information using informal signals such as emotional and pragmatic cues. Timing is crucial in these scenarios because adjusting to fast changing circumstances requires promptly updated situation awareness, which can only be achieved if all the actors in the network maintain an appropriate communications tempo using informal communicative processes shared with all the other actors. ${ }^{55}$ To sum up, in hybrid warfare the success of both action and decision depends on effective, parsimonious, and timely team communication, which in turn involves anthropomorphic cues.

Informal communication between human operatives and AAAs is both a desirable and realistic outcome and depends on basic mechanisms of social cognition. Hence, artificial agents can be effectively employed in military operations only factoring in both the desirable and the undesired implications of anthropomorphism on a team's constituent parts. Social cognition research suggests that anthropomorphism is a basic feature of human psychology and, as such, it represents an ineliminable component of HAI. That is why social robotics research systematically studies the roots of anthropomorphism to maximize human-robot coordination through anthropomorphic design, taking advantage of how AAAs are generally perceived by humans to improve the quality of the HAI experience. ${ }^{56}$

That is why pro-actively integrating anthropomorphic design motives (instead of accepting them as an accidental byproduct) should be seen as a necessary stage in the roadmap

\footnotetext{
55 The Actor-Network-Theory can be profitably applied to our investigation. The theory considers both humans and AAAs as "actants" whose role defines, and at the same time is defined, by the particular networks in which they operate. The relation between individual actants and network's objectives has to be understood systemically, as their performance can only be assessed against the system's objectives See S. Soltanzadeh, M. Mooney, "Players Within a Team: Understanding the Structure of Team Performance Through Individual Functions and Team Objectives," International Journal of Sports Science o Coaching 5/1 (2018): 84-89. If the hybrid unit's dynamics (leadership, agency, and the cognitive burden of decision) are intelligible it is because they are informed by the roles taken up by human and robotic actants, which in turn are importantly shaped by the phenomenon of anthropomorphism. In the military context, each network represents a unit, in which humans and AAAs cooperate to achieve the same tactical objectives. Unit's effectiveness depends on the HAI's robustness and fluidity. Hence, anthropomorphism can be used to re-design and optimize network relations within the unit.

56 B.R. Duffy, "Anthropomorphism and the Social Robot," Robotics and Autonomous Systems 42/3 (2003): 177-90; C. Bartneck, D. Kulić, E. Croft, S. Zoghbi, "Measurement Instruments for the Anthropomorphism, Animacy, Likeability, Perceived Intelligence, and Perceived Safety of Robots," International Journal of Social Robotics 1/1 (2009): 71-81.
} 
to the development of efficacious military AAAs. This implies that AAAs design and troops training should be designed in parallel, aiming towards the development of natural interfaces to optimize human-machine communication, as this could significantly reduce the costs and times necessary to make hybrid military teams operation ready. Considering the limitations in the development of generalistic AI, specific studies are required to maximize the benefits of anthropomorphism in task-specific AAAs in accord with their distinctive design requirements.

\section{Funding acknowledgement}

This material is based upon work supported by the Air Force Office of Scientific Research and the Minerva Initiative under award number FA9550-18-1-0039. The work has also received the support of the Australian Government.

\section{Disclosure statement}

The authors declare that they have no conflict of interest.

\section{References}

Abbass, H.A., Scholz, J., Reid, D.J. "Foundations of Trusted Autonomy: An Introduction." In Foundations of Trusted Autonomy, edited by Hussein A. Abbass, Jason Scholz, Darryn J. Reid, 1-12. New York: Springer, 2018. https://doi.org/10.1007/978-3-319-64816-3_1.

Airenti, G. "The Cognitive Bases of Anthropomorphism: From Relatedness to Empathy." International Journal of Social Robotics 7/1 (2015): 117-27. https://doi.org/10.1007/s12369014-0263-x.

-. "The Development of Anthropomorphism in Interaction: Intersubjectivity, Imagination, and Theory of Mind." Frontiers in Psychology 9 (November 2018): 2136. https://doi.org/10.3389/fpsyg.2018.02136.

Barnes, M., Evans, A.W. “Soldier-Robot Teaming: An Overview.” In Human-Robot Interactions in Future Military Operations, edited by M. Barnes and F. Jentsch. Farnham: Ashgate Publishing, 2010.

Barnes, M.J., Jentsch, F.J. (eds.). Human-Robot Interactions in Future Military Operations. Farnham: Ashgate Publishing, 2010. https://doi.org/10.4324/9781315587622.

Bartneck, C., Kulić, D., Croft, E., Zoghbi, S. "Measurement Instruments for the Anthropomorphism, Animacy, Likeability, Perceived Intelligence, and Perceived Safety of Robots." International Journal of Social Robotics 1/1 (2009): 71-81. https://doi.org/ 10.1007/s12369-008-0001-3. a

Bartneck, C., Kanda, T., Mubin, O., Al Mahmud, A. "Does the Design of a Robot Influence Its Animacy and Perceived Intelligence?" International Journal of Social Robotics 1 (2009): 195-204. https://doi.org/10.1007/s12369-009-0013-7.

Breazeal, Cynthia. "Towards Sociable Robots." Robotics and Autonomous Systems 42/3-4 (2003): 167-175.

Breazeal, C., Dautenhahn, K., Kanda, T. 2016. "Social Robotics." Springer Handbook of Robotics. https://doi.org/10.1007/978-3-319-32552-1_72.

Cappuccio, M.L. “Inference or Familiarity?”' Synthesis Philosophica 58/2 (2015): 253-72.

Cappuccio, M.L., Peeters, A., McDonald, W. "Sympathy for Dolores: Moral Consideration for Robots Based on Virtue and Recognition.” Philosophy \& Technology 33 (2020) : 9-31. https://doi.org/10.1007/s13347-019-0341-y. 
Cappuccio, M.L., Wheeler, M. "When the Twain Meet: Could the Study of Mind Be a Meeting of Minds?” In Postanalytic and Metacontinental: Crossing Philosophical Divides, edited by Jack Reynolds, James Chase, Ed Mares, James Williams, 125-44. London: Continuum, 2010.

Cappuccio, M.L, Wheeler, M. "Ground-Level Intelligence: Inter-Context Frame Problem and Dynamics of the Background." In Knowing without Thinking. Mind, Action, Cognition and the Phenomenon of the Background, edited by Zdravko Radman. Basingstoke: Palgrave Macmillan, 2012.

Carpenter, J., Eliot, M., Schultheis, D. “The Uncanny Valley: Making Human-Nonhuman Distinctions." In Proceedings of the 5th International Conference on Cognitive Science (2006): 8182. http://www.academia.edu/download/30609409/p81.pdf.

Carpenter, J. "Just Doesn't Look Right: Exploring the Impact of Humanoid Robot Integration into Explosive Ordnance Disposal Teams." In Handbook of Research on Technoself: Identity in a Technological Society, edited by Rocci Luppicini, 609-36. Hershey: IGI Global, 2013.

Chen, J.Y., Anthony, C., Selkowitz, R., Stowers, K., Lakhmani, S.G., Barnes, M.J. 2017. "Human-Autonomy Teaming and Agent Transparency." Proceedings of the Companion of the 2017 ACM/IEEE International Conference on Human-Robot Interaction - HRI '17. https://doi.org/10.1145/3029798.3038339.

Chen, J.Y., Quinn, S., Wright, J., Barnes, M., Barber, D., Adams, D. "Human-Agent Teaming for Robot Management in Multitasking Environments." 2013 8th ACM/IEEE International Conference on Human-Robot Interaction (HRI) (2013). https://doi.org/10.1109/ hri.2013.6483522.

Cosenzo, K.A., Barnes, M. J. "Human-Robot Interaction Research for Current and Future Military Applications: From the Laboratory to the Field." Unmanned Systems Technology XII (2010). www.spiedigitallibrary.org/conference-proceedings-of-spie/7692/769204/Hum an-robot-interaction-research-for-current-and-future-military-applications/10.1117/12.8 50038.short?casa_token=-v4XTIBdAosAAAAA:mVxNaDQmnNpXpC2NI8Q-rBqfVI smBySfb4eEzn5OXR5FUf1Kdk0ADHDde1BEgBGb8iJk5G1hyQ.

Dacey, M. “Anthropomorphism as cognitive bias.” Philosophy of Science 84:5 (2017): 1152-1164.

Cummings, M.L. "Artificial Intelligence and the Future of Warfare." International Security Department and US and the Americas Programme, Chatham House - The Royal Institute of International Affairs (January 2017).

Damiano, L., Dumouchel, P. "Anthropomorphism in Human-Robot Co-Evolution." Frontiers in Psychology 9 (2018): 117. https://doi.org/10.3389/fpsyg.2018.00468 .

Dautenhahn, K. "Socially Intelligent Robots: Dimensions of Human-Robot Interaction." Philosophical Transactions of the Royal Society of London. Series B, Biological Sciences 362 (2007): 679-704.

DiSalvo, C., Gemperle, F. "From Seduction to Fulfillment: The Use of Anthropomorphic Form in Design." In Proceedings of the 2003 International Conference on Designing Pleasurable Products and Interfaces (2003): 67-72. https://doi.org/10.1145/782896.782913.

DiSalvo, C., Hanington, B., Forlizzi, J. “An Accessible Framework of Emotional Experiences for New Product Conception." In Design and Emotion, edited by Deana McDonagh, Paul Hekkert, Jeroen van Erp, Diane Gyi. Boca Raton: CRC Press, 2003.

Duffy, B.R. "Anthropomorphism and the Social Robot." Robotics and Autonomous Systems 42/3 (2003): 177-90. https://doi.org/10.1016/S0921-8890(02)00374-3.

Epley, N., Waytz, A., Cacioppo, J. T. "On Seeing Human: A Three-Factor Theory of Anthropomorphism.” Psychological Review 114/4 (2007): 864-86. https://doi.org/10.1037/ 0033-295X.114.4.864. 
Epley, N., Waytz, A., Akalis, S., Cacioppo, J.T. "When We Need A Human: Motivational Determinants of Anthropomorphism." Social Cognition 26/2 (2008): 143-55. https://doi.org/10.1521/soco.2008.26.2.143.

Eyssel, F.A., Pfundmair, M. "Predictors of Psychological Anthropomorphization, Mind Perception, and the Fulfillment of Social Needs: A Case Study with a Zoomorphic Robot" 2015 24th IEEE International Symposium on Robot and Human Interactive Communication (2015). https://doi.org/10.1109/roman.2015.7333647.

Foster Thompson, L., Gillan, D.J. “Social Factors in Human-Robot Interaction.” In HumanRobot Interactions in Future Military Operations, edited by Barnes and Jentsch, 67-81. Boca Raton: CRC Press, 2017.

Gallagher, S. "Understanding Others: Embodied Social Cognition.” In Handbook of Cognitive Science, edited by Paco Calvo and Antoni Gomila, 437-52. San Diego: Elsevier, 2008.

- "Inference or Interaction: Social Cognition without Precursors." Philosophical Explorations: An International Journal for the Philosophy of Mind and Action 11/3 (2008): 16374. https://doi.org/10.1080/13869790802239227.

Gallese, V. "The Shared Manifold Hypothesis: Embodied Simulation and its Role in Empathy and Social Cognition." In Empathy in Mental Illness, edited by Tom F. D. Farrow, Peter W. R. Woodruff, Tom F. D. Farrow, and Peter W. R. Woodruff, 448-72. Cambridge: Cambridge University Press, 2007. https://doi.org/10.1017/CBO9780511543753.025.

Galliot, J.C. "Uninhabited systems in the civilian realm: Some ethical concerns." IEEE Technology and Society Magazine 31 (2012): 13-16. http://dx.doi.org/10.1109/MTS.2012. 2202017.

—. "Unmanned Systems and War's End: Prospects for Lasting Peace." Dynamiques Internationale 8 (2013): 1 - 24.

—. Military Robots: Mapping the Moral Landscape. New York: Routledge, 2015.

—_. "Defending Australia in the digital age: toward full spectrum defence." Defence Studies 16 (2016): 157 - 175. http://dx.doi.org/10.1080/14702436.2016.1165596.

—. "The Soldier's Tolerance for Autonomous Systems." Paladyn, Journal of Behavioral Robotics 9/1 (2018): 124-36. https://doi.org/10.1515/pjbr-2018-0008.

Harrison, M.A., Hall. A.E. "Anthropomorphism, Empathy, and Perceived Communicative Ability Vary with Phylogenetic Relatedness to Humans." Journal of Social, Evolutionary \& Cultural Psychology 4/1 (2010): 34. https://doi.org/10.1037/h0099303.

Heijnen, S., de Kleijn, R., Hommel, B. "The Impact of Human-Robot Synchronization on Anthropomorphization." Frontiers in Psychology 9 (2018): 2607. https://doi.org/10.3389 /fpsyg.2018.02607.

Hill, S.G., Barber, D., W. Evans, A. "Achieving the Vision of Effective Soldier-Robot Teaming." Proceedings of the Tenth Annual ACM/IEEE International Conference on HumanRobot Interaction Extended Abstracts - HRI'15 Extended Abstracts (2015). https://doi.org/10. 1145/2701973.2702026.

Hoffman, G., and Breazeal, C. "Collaboration in Human-Robot Teams." In ALAA 1st Intelligent Systems Technical Conference. Infotech@Aerospace Conferences. American Institute of Aeronautics and Astronautics (2004). https://doi.org/10.2514/6.2004-6434.

—. "Effects of Anticipatory Action on Human-Robot Teamwork Efficiency, Fluency, and Perception of Team." Proceedings of the ACM/IEEE International (2007). https://dl.acm.org/citation.cfm?id=1228718.

Hoffman, G. Ensemble: Fluency and Embodiment for Robots Acting with Humans. Ph.D. Media Arts and Sciences. MIT, September 2007.

—. "Embodied Cognition for Autonomous Interactive Robots." Topics in Cognitive Science 4/4 (2012): 759-72. https://doi.org/10.1111/j.1756-8765.2012.01218.x. http://ir.canterbury.ac.nz/handle/10092/8698. 
Lendon, B. "Australian military gets first drone that can fly with artificial intelligence." CNN (2020). Retrieved at https://edition.cnn.com/2020/05/05/asia/australia-loyal-wingmandrone-intl-hnk/index.html.

Kaplan, A.D., Sanders, T., Hancock, P.A. "The Relationship Between Extroversion and the Tendency to Anthropomorphize Robots: A Bayesian Analysis." Frontiers in Robotics and AI 5 (2019): 135. https://doi.org/10.3389/frobt.2018.00135.

Lin, P., Mehlman, M., Abney, K., Galliott, J. "Super Soldiers (Part 1): What is Military Human Enhancement?" In Global Issues and Ethical Considerations in Human Enhancement Technologies, edited by S. Thompson, 119 - 138. Hershey: IGI Global, 2014.

Lin, P., Mehlman, M., Abney, K., Vallor, S., French, S., Galliott, J., Burnam-Fink, M., LaCroix, A., Schuknecht S. "Super Soldiers (Part 2): The Ethical, Legal, and Operational Implications." In Global Issues and Ethical Considerations in Human Enhancement Technologies, edited by S. Thompson, 139 - 160. Hershey: IGI Global, 2014.

Mori, M. “The Uncanny Valley.” Energy 7/4 (2005): 33-35.

Mutlu, B., Bartneck, C., Ham, J., Evers, V., Kanda, T. (eds). Social Robotics: Third International Conference on Social Robotics, ICSR 2011, Amsterdam, The Netherlands, November 24-25, 2011. Proceedings. New York: Springer, 2011.

Nyholm, S. "The Ethics of Crashes with Self-Driving Cars: A Roadmap." Philosophy Compass 13/7 (2018). https://doi.org/10.1111/phc3.12506.

Obaid, M., Kistler, F., Kasparavičiūtè, G., Yantaç, A.Y., and Fjeld, M. "How Would You Gesture Navigate a Drone?: A User-Centered Approach to Control a Drone." In Proceedings of the 20th International Academic Mindtrek Conference, 113-21. New York: Association for Computing Machinery, 2016. https://doi.org/10.1145/2994310. 2994348.

Ono, T., Imai, M., Ishiguro, H. "Anthropomorphic Communications in the Emerging Relationship between Humans and Robots." Proceedings 9th IEEE International Workshop on Robot and Human Interactive Communication (2000). https://doi.org/10.1109/roman.20 00.892519 .

Reichenbach, J., Bartneck, C., Carpenter, J. "Well Done, Robot! The Importance of Praise and Presence in Human-Robot Collaboration." In ROMAN 2006 - The 15th IEEE International Symposium on Robot and Human Interactive Communication, 86-90. New Jersey: IEEE, 2006. https://doi.org/10.1109/ROMAN.2006.314399.

Riek, L. D., Rabinowitch, T., Chakrabarti, B., Robinson, P. "How Anthropomorphism Affects Empathy toward Robots." In 2009 4th ACM/IEEE International Conference on Human-Robot Interaction (HRI) (2009): 245-46. https://doi.org/10.1145/1514095.1514158.

Salem, M., Eyssel, F., Rohlfing, K., Kopp, S., and Joublin, F. "Effects of Gesture on the Perception of Psychological Anthropomorphism: A Case Study with a Humanoid Robot.” In Social Robotics, 31-41. Berlin/Heidelberg: Springer, 2011. https://doi.org/ 10.1007/978-3-642-25504-5_4.

Scharre, P. Army of None: Autonomous Weapons and the Future of War. New York: WW Norton \& Company, 2018.

Servais, V. "Anthropomorphism in Human-Animal Interactions: A Pragmatist View." Frontiers in Psychology 9 (December 2018): 2590. https://doi.org/10.3389/fpsyg.2018.0 2590.

Severson, R.L., and Shailee, R.W. "Imagining Others' Minds: The Positive Relation Between Children's Role Play and Anthropomorphism." Frontiers in Psychology 9 (November 2018): 2140. https://doi.org/10.3389/fpsyg.2018.02140.

Shen, Solace, Slovak, Petr, Jung, Malte F. “"Stop. I See a Conflict Happening.": A Robot Mediator for Young Children's Interpersonal Conflict Resolution." HRI '18: Proceedings of the 2018 ACM/IEEE International Conference on Human-Robot Interaction (2018): 69-77. 
Singer, P.W. Wired for War: The Robotics Revolution and Conflict in the 21st Century. London: Penguin, 2009.

Slocombe, G. "Autonomous Warrior: Major Air, Land and Sea Exercise." Asia-Pacific Defence Reporter (September 26, 2019). Retrieved on May 1, 2019 from https://asiapacificdefen cereporter.com/autonomous-warrior-2018/.

Soltanzadeh, S., and Mooney, M. "Players Within a Team: Understanding the Structure of Team Performance Through Individual Functions and Team Objectives." International Journal of Sports Science \& Coaching 5/1 (2018): 84-89. https://doi.org/10.1123/iscj.20170032.

Sparrow, R., and Howard, M. "When Human Beings Are like Drunk Robots: Driverless Vehicles, Ethics, and the Future of Transport." Transportation Research Part C: Emerging Technologies 80 (July 2017): 206-15. https://doi.org/10.1016/j.trc.2017.04.014.

Sparrow, R. "Robots and Respect: Assessing the Case Against Autonomous Weapon Systems." Ethics \& International Affairs 30/1 (2016): 93-116. https://doi.org/10.10 17/S0892679415000647.

Swiderska, A., and Küster, Dennis. "Avatars in Pain: Visible Harm Enhances Mind Perception in Humans and Robots." Perception 47/12 (2018). https://doi.org/10.1177/ 0301006618809919 .

Tahiroglu, D., and Taylor, M. “Anthropomorphism, Social Understanding, and Imaginary Companions." The British Journal of Developmental Psychology 37/2 (November 2018): 284299. https://doi.org/10.1111/bjdp.12272.

Tucker, P. “An AI Just Beat a Human F-16 Pilot in a Dogfight - Again.”. Defense One online (August 20, 2020). Retrieved at https://www.defenseone.com/technology/2020/08/aijust-beat-human-f-16-pilot-dogfight-again/167872/.

Turnbull, G. "Why the British army tested robots in muddy fields." C4ISRnet (January 11, 2019).

Urquiza-Haas, E.G., Kotrschal, K. "The Mind behind Anthropomorphic Thinking: Attribution of Mental States to Other Species." Animal Behaviour 109 (November 2015): 167-76. https://doi.org/10.1016/j.anbehav.2015.08.011.

Vertesi, J. "Seeing like a Rover: Visualization, Embodiment, and Interaction on the Mars Exploration Rover Mission." Social Studies of Science 42/3 (2012): 393-414. https://doi.org/10.1177/0306312712444645.

Waytz, A., Cacioppo, J.T., Epley, N. "Who Sees Human? The Stability and Importance of Individual Differences in Anthropomorphism." Perspectives on Psychological Science 5/3 (2014): 219-232.

Waytz, A., Epley, N., and Cacioppo. J. T. "Social cognition unbound: Insights into anthropomorphism and dehumanization." Current Directions in Psychological Science 19/1 (2010): 58-62.

White, R.C., Remington, A. "Object Personification in Autism: This Paper Will Be Very Sad If You Don't Read It.” Autism: The International Journal of Research and Practice 23/4 (2018). https://doi.org/10.1177/1362361318793408.

Wodehouse, A., Brisco, R., Broussard, E., Duffy, A. "Pareidolia: characterizing facial anthropomorphism and its implications for product design." Journal of Design Research, 16/2 (2018): 83-98.

Zawieska, K. n.d. "Do Robots Equal Humans? Anthropomorphic Terminology in LAWS." 2015 UN CCW Informal Meeting of Experts on Lethal Autonomous Weapons Systems at Geneva, Switzerland (2015).

"Washington Post 2007 Bots on The Ground.pdf." n.d. 
Zlotowski, J.A. "Understanding Anthropomorphism in the Interaction between Users and Robots." Doctoral Thesis. University of Canterbury, 2015. http://ir.canterbury.ac.nz/ handle/10092/11259.

Złotowski, J.A., Proudfoot, D., Yogeeswaran, K., Bartneck, C. "Anthropomorphism: Opportunities and Challenges in Human-Robot Interaction." Advanced Robotics: The International Journal of the Robotics Society of Japan 7/3 (2015): 347-60. https://doi.org/10.1007/s12369-014-0267-6.

Złotowski, J.A, Sumioka, H., Bartneck, C., Nishio, S., Ishiguro, H. "Understanding Anthropomorphism: Anthropomorphism Is Not a Reverse Process of Dehumanization." In Social Robotics, edited byAbderrahmane Kheddar, Eiichi Yoshida, Shuzhi Samk GeKenji Suzukik John-John Cabibihan, Friederike Eyssel, Hongsheng He, 618-27. New York: Springer International Publishing, 2017. doi.org/10.1007/978-3-319-70022-9_61.

Złotowski, J.A, Sumioka, H., Eyssel, F., Nishio, S., Bartneck, C., Ishiguro, H. 2018. "Model of Dual Anthropomorphism: The Relationship Between the Media Equation Effect and Implicit Anthropomorphism." International Journal of Social Robotics 10 (2018): 701-714. https://doi.org/10.1007/s12369-018-0476-5.

Zlotowski, J.A., Sumioka, H., Nishio, S., Glas, D.F., Bartneck, C., Ishiguro, H. "Persistence of the Uncanny Valley: The Influence of Repeated Interactions and a Robot's Attitude on Its Perception.” Frontiers in Psychology 6 (2015): 883. https://www.frontiersin.org/articles/ 10.3389/fpsyg.2015.00883.

Zlotowski, J., Proudfoot, D., Bartneck, C. "More Human than Human: Does the Uncanny Curve Really Matter?" Proceedings of the HRI2013 Workshop on Design of Humanlikeness in HRI from uncanny valley to minimal design (Tokyo, 2013): 7-13. 